\title{
LA SEPARACIÓN ENTRE PALABRAS EN LA ESCRITURA DE NIÑOS QUE INICIAN LA ESCOLARIDAD PRIMARIA*
}

\author{
WORD SEPARATION IN THE WRITING OF CHILDREN BEGINNING PRIMARY SCHOOL
}

\author{
Maira Querejeta** ${ }^{* *}$ Telma Piacente***, Bárbara Guerrero Ortiz-Hernán**** ${ }^{*}$ \\ Elda Alicia Alva Canto*****
}

\begin{abstract}
*Los datos utilizados en este trabajo forman parte de la Tesis Doctoral La separación entre palabras en la escritura de oraciones y textos de la primera autora. Este proyecto fue financiado por el Consejo Nacional de Investigaciones Científicas y Técnicas (CONICET, Argentina) y la Red de Macrouniversidades de América Latina y el Caribe.

**Doctora en Psicología. Becaria Posdoctoral del Consejo Nacional de Investigaciones Científicas y Técnicas (CONICET). E-Mail: mairaquerejeta@gmail.com

Diagonal $74 \mathrm{~N}^{\circ}$ 1312, (1900) La Plata, Provincia de Buenos Aires. República Argentina. ***Psicóloga Clínica. Directora del Instituto de Psicología de la Facultad de Psicología de la Universidad Nacional de La Plata (UNLP).

****Licenciada en Psicología. Becaria Doctoral del Laboratorio de Infantes en la Facultad de Psicología de la Universidad Nacional Autónoma de México (UNAM).

*****Doctora en Psicología General Experimental (UNAM). Directora del Laboratorio de Infantes de la Facultad de Psicología de la Universidad Nacional Autónoma de México
\end{abstract} (UNAM).

\section{RESUMEN}

En la escritura convencional de oraciones y de textos, las palabras están separadas por espacios en blanco. Sin embargo, en los inicios de la alfabetización suelen observarse hiposegmentaciones e hipersegmentaciones entre palabras. Las investigaciones sobre el tema son escasas, limitándose a registrar su aparición, sin llegar a establecer todos los tipos de uniones o separaciones. En función de ello, a partir de un diseño descriptivo transeccional correlacional, el propósito de este trabajo fue caracterizar el tipo y frecuencia de separaciones no convencionales entre palabras que aparecen durante el primer año de escolaridad en niños argentinos y mexicanos con expectativas de logro diferentes. En segundo lugar, examinar las posibles relaciones que guar- dan esas separaciones con el desempeño lector y el nivel de vocabulario. Para ello, se examinaron 30 niños argentinos y mexicanos que tenían entre 6 y 7 años de edad mediante pruebas de escritura, lectura y vocabulario. En el análisis lingüístico de las producciones infantiles se utilizó la noción gramatical de formante morfológico que posibilitó caracterizar la totalidad y distribución de las segmentaciones no convencionales. Los resultados más significativos aportan evidencia empírica de la presencia de tales segmentaciones en los niveles iniciales del aprendizaje y una distribución semejante del tipo de errores cometidos, independientemente de la nacionalidad y exigencias en la alfabetización. Se concluye, con las limitaciones del caso en razón del número de niños incluidos, que parecen constituir una etapa evolutiva, a ser considerada en fu- 
turas investigaciones y en estrategias de enseñanza de la escritura de oraciones y textos.

Palabras clave: Niños; Escritura; Separación entre palabras; Hiposegmentación; Hipersegmentación.

\section{ABSTRACT}

In conventional writing of sentences and texts, the words are separated by blank spaces. However, the beginnings of literacy often observed hyposegmentations and hyper-segmentations between words. Hypo-segmentation occurs when adequate separation between words is missing, whereas hyper-segmentation takes place when a word is separated incorrectly and a space is inserted between two of its elements. Research on this topic is scarce and has registered the appearance of arbitrary joints and separations both in content and functional words, but has failed to account for all of the phenomena observed in children's writing. This paper has two aims: first of all, characterize the type and frequency of the unconventional separations between words written at the end of the first year of primary schooling in Mexican and Argentinian children, by reason of the different literacy modalities of both countries. The expectations prescribed in the curricula of both countries are different: Mexico expected children to learn to read and write words and sentences at the end of preschool. In Argentina those expectations are related to the first cycle of primary schooling. Regarding the teaching methodologies to which the children screened were exposed, although it is not possible to characterize them as exhaustive, since teachers often introduce different variants, they are predominantly phonic in the case of Mexico, and derived from the psychogenesis of the language written in the case of Argentina. Second, identifying the possible connection between these phenomena and variables as reading, writing and vocabulary performance range. Therefore, thirty Argentinian and Mexican children between six and seven years of age (mean age: six years and nine months) were examined through (both spontaneous and dictated) writing, reading and vocabulary tests. The notion of morphological for- mants was used to linguistic analysis of child productions; this allowed for the categorization of all the unconventional segmentations which came up. Among some of the results of this study, we would like that the proposed classification turned out to be extremely useful and even more adequate than other classifications in terms of word -function and word -content. Secondly, it should be pointed out that unconventional separations were more frequently observed in dictations. Thirdly, Mexican children obtained higher scores when reading words and pseudo-words, which demonstrate that they have better control of the alphabetic principle in comparison with the Argentinian ones. Fourthly, and in agreement with other research, hypo-segmentation frequency of appearance turned out to be higher than hyper-segmentation, both groups displaying similar characteristics. On the one hand, the average scores for the mistakes made failed to show any significant differences in terms of children's nationalities. On the other hand, even though such hypo-segmentations mostly appeared due to union of a lexical and a grammatical formant, in other cases only lexical or grammatical formants were joined, which proves once again how useful the categorization was. Finally, correlations with the levels of word reading and writing, reading comprehension and vocabulary range turned out to be low. In connection with this, with the limitations of the case number, it can be pointed out that wrong segmentation goes beyond the children's performance within such variables and seems to be an evolutive stage within the progressive control of reflection upon and conscious manipulation of lexical items. It would prove interesting to increase the study to one larger number of participants and made longitudinal studies, during the schooling process enabling to clear out the evolution of the phenomena examined throughout the schooling period. It should be pointed out that unconventional word separations are not always spotted at school and that their persistence, unless proper intervention strategies are used, may have subsequent repercussions on text production, which corresponds to more advanced schooling levels.

Key words: Children; Writing; Word segmentation; Hypo-segmentation; Hyper-segmentation. 


\section{INTRODUCCIÓN}

La escritura convencional de oraciones y textos implica no sólo alcanzar competencias en la escritura de palabras utilizando estrategias analíticas completas (Ball \& Blachman, 1991), sino también el dominio de las convenciones ortográficas de una lengua, es decir, las normas que rigen el uso de determinadas letras para representar ciertos fonemas, de mayúsculas, minúsculas y tildes, de signos de puntuación, de las normas de separación silábica para la división correcta de una palabra y de la separación ortográfica de las palabras por medio de espacios en blanco (Linares Rivas, 2000; RAE $\&$ ASALE, 2010).

El propósito del trabajo que se informa fue caracterizar el tipo y frecuencia de separaciones no convencionales entre palabras en niños argentinos y mexicanos, con diferentes expectativas de logro y consecuentemente modalidades de alfabetización, a la finalización del primer año de la escolaridad primaria. Tales expectativas para las edades consideradas, prescriptas en las currícula de ambos países son diferentes: en México se espera que los niños aprendan a leer y escribir palabras y oraciones a la finalización de la preescolaridad (SEP, 2011a, 2011b). En Argentina esas expectativas refieren al primer ciclo de la escolaridad primaria (DGCE, 2008).

En cuanto a la metodología de enseñanza a la que estuvieron expuestos los niños examinados, se trata en ambos casos del aprendizaje de la lectura y la escritura en un sistema de escritura alfabético. Si bien no es posible caracterizarlas de modo exhaustivo, dado que los maestros suelen introducir distintas variantes, aluden prevalentemente a métodos fónicos en el caso de México (SEP, 2011b) y a métodos derivados de la psicogénesis de la lengua escrita en el caso de Argentina (Molinari \& Corral, 2008).

En los métodos fónicos, para la identificación y codificación de palabras se pone más énfasis en el dominio progresivo del principio alfabético, que requiere aprender cómo se segmenta el habla en los sonidos (fonemas), el conocimiento de los grafemas (letras) que los representan y la aplicación de las reglas de correspondencia entre ambos. Dicho de otro modo, en la asociación letra sonido más que en el reconocimiento total de la palabra u otras claves basadas en el significado (Adams, 1990; Cook Moats, 2010; Marder, 2008; Snow \& Juel, 2005).

En los métodos derivados del enfoque psicogenético se sostiene que en la adquisición de la escritura se producen sucesivas estructuraciones y reestructuraciones generadas por contradicciones entre distintos esquemas implicados en un mismo momento del proceso o por conflictos entre los esquemas y lo real (Ferreiro \& Teberosky, 1979).

Desde esta perspectiva, el proceso de conceptualización de la escritura se caracteriza por la construcción de sucesivas formas de diferenciación de los aspectos cuantitativos y cualitativos del sistema de escritura. En tal sentido, el niño formula diversas hipótesis referidas al modo de funcionamiento de dicho sistema. Entre ellas, una hipótesis silábica que lo lleva a representar cada sílaba mediante una grafía. Esta hipótesis cede paulatinamente su lugar a un análisis que va más allá de la sílaba, cuando los conflictos con los que se enfrenta el niño lo obligan a abandonarla. Estos conflictos son el resultado de una puja entre los resultados obtenidos (grafía), la conceptualización actual del sujeto y las escrituras producidas por los otros, en las que está implícita la práctica social, o sea la escritura convencional (la norma). De esta manera se pasa a una etapa de transición denominada silábico - alfabética hasta que el niño elabora la hipótesis alfabética que lo lleva a representar cada sonido mediante una grafía (Ferreiro \& Teberosky, 1979).

Cuando se trasciende la escritura de palabras, es decir cuando se trata de oraciones $\mathrm{y}$ de textos, un aspecto que ha sido insuficientemente examinado refiere a la separación entre palabras o segmentación lexical; este fue el objetivo principal de este trabajo. Esta separación consiste en la habilidad para discriminar los límites de las pala- 
bras (Gombert, 1990). Esta tarea no resulta sencilla en los primeros tramos del proceso de alfabetización, incluso para aquellos niños que dominan el principio alfabético. De ahí que suelen aparecer frecuentemente segmentaciones lexicales no convencionales de las palabras escritas. Dos tipos de errores son posibles: hipo e hipersegmentaciones. La hiposegmentación ocurre cuando no aparecen separaciones entre palabras. En cambio, la hipersegmentación se produce cuando se separa indebidamente una palabra a través de un espacio entre dos de sus elementos (Correa \& Dockrell, 2007).

Si bien en las últimas décadas se han multiplicado las investigaciones acerca del aprendizaje del lenguaje escrito, el número de estudios que ha analizado la capacidad para segmentar frases en palabras es escaso (Báez, 1999; Borzone de Manrique \& Massone, 1987; Correa, 2005; Correa \& Dockrell, 2007; Gombert, 1990; Piacente, 2005; Tolchinsky, 2006). En ellos no se alcanza a describir y examinar en detalle la frecuencia y los patrones de errores de las segmentaciones no convencionales en las producciones infantiles, ni a explicarlos de manera satisfactoria.

Las investigaciones realizadas con niños de distintas edades señalan la presencia de segmentaciones lexicales no convencionales en las producciones escritas espontáneas. Entre ellas, Báez (1999) presentó evidencia acerca de la preponderancia de hiposegmentaciones, variando los porcentajes en función del nivel escolar. En su trabajo observó que las segmentaciones arbitrarias aparecieron en mayor medida entre los niños de segundo y tercer grado y en menor medida, entre los de grados superiores, aún cuando se observaron incluso en séptimo grado.

A su vez, Ferreiro y Pontecorvo (1996) informan la presencia de segmentaciones no convencionales en la escritura infantil de niños hablantes de español, italiano y portugués, siendo mayor la frecuencia de hiposegmentaciones que de hipersegmentaciones. Destacan que los niños presentan problemas para separar las preposiciones, artículos y conjunciones respecto de las palabras de contenido como sustantivos, verbos o adjetivos (Ferreiro \& Pontecorvo, 1996; Tolchinsky, 2006). Estas características, según las autoras, son esperables dado que las últimas típicamente tienen referentes delimitados en algún contexto, mientras que las primeras sólo pueden ser interpretadas en relación con las oraciones en las que aparecen.

En otro trabajo, Correa y Dockrell (2007) examinaron los cambios en el desarrollo de la habilidad para definir los límites de las palabras escritas en niños de habla portuguesa que se encontraban en las fases tempranas de la escolaridad formal, hallando que las medias en lectura de palabras, conciencia morfológica y vocabulario descendían en los niños que presentaban alta frecuencia de hiposegmentaciones e hipersegmentaciones. Afirman que "althought both hyposegmentation and hypersegmentation were related to lower levels of reading and morphological awareness occurrences of hypersegmentation where limited to the initial stages of education" (p. 828). Estos resultados sugieren que los avances a través de la alfabetización y el lenguaje contribuyen a la comprensión de los patrones de segmentación de palabras. Para estas autoras, la sensibilidad de los niños a la gramática, especialmente a la morfología, y el incremento del vocabulario juegan un papel importante en el desarrollo de la habilidad para segmentar las palabras en las oraciones y textos escritos. En tal sentido argumentan que para segmentar convencionalmente, los niños se guían por su sensibilidad a la morfología, ya sea en la separación de una palabra - función de la palabra de contenido más cercana en una frase o por el mantenimiento de la integridad de una palabra que comenzó con una sílaba que puede funcionar como una palabra - función. Por otra parte, sostienen que el conocimiento del vocabulario proporciona tanto información fonológica como morfológica, de modo tal que los niños con un vocabulario más amplio tienen representaciones fonológicas más detalladas de las palabras, que apoyan a la ortografía de dos maneras diferentes: mediante la mejora de la con- 
ciencia fonológica y la disponibilidad de la forma fonológica de una palabra que debe ser escrita. En tal sentido las investigaciones de Erhi $(1997,1998)$ plantean que la amalgama entre ortografía, pronunciación y significado formada en la memoria a largo plazo para leer palabras también es útil para ortografiarlas.

En un estudio realizado con niños de 6 a 11 años de edad que concurrían a escuelas de diferente procedencia sociocultural (Querejeta, 2011) se observaron fenómenos de hiper e hiposegmentación en la mayoría de los alumnos en proceso de alfabetización. Tales fenómenos aparecieron en las producciones infantiles de hasta cuarto y quinto año de la escolaridad primaria, disminuyendo de manera estadísticamente significativa en sexto grado $^{1}$.

De acuerdo a los antecedentes descriptos previamente, las segmentaciones no convencionales parecen constituir una característica en el desarrollo de la escritura infantil. En tal sentido, aparecen prevalentemente en los primeros años escolares y en aquellos casos en los que los procesos de enseñanza y aprendizaje parecen ser menos exitosos. Sin embargo, su caracterización no resulta exhaustiva.

En razón de ello, este trabajo fue destinado en primer lugar, a identificar y comparar las características y frecuencia que asumen las segmentaciones no convencionales que aparecen en las escrituras de niños argentinos y mexicanos a la finalización del primer año de escolaridad, en razón de los diferentes objetivos que se propone la alfabetización inicial. Para ello ha sido necesario identificar en principio el tipo de hiper e hiposegmentaciones que aparecen en el período considerado de la escolaridad formal, a partir de la clasificación a la que se arribara en

1 Cabe señalar que en poblaciones provenientes de sectores pobres se presentaron otras dificultades, particularmente en relación con la comprensión textual y la producción escrita. un trabajo anterior (Querejeta, 2011). Se entiende por ello la disposición de los fenómenos bajo estudio a partir de los atributos comunes que presentan (RAE, 2010) las uniones y separaciones no convencionales, tomando en consideración la noción gramatical de formante morfológico (Di Tullio, 1999). En segundo lugar, una vez caracterizadas las separaciones no convencionales, a indagar si existen relaciones entre estos fenómenos y el desempeño en lectura, escritura y vocabulario, variables estas últimas examinadas a través de pruebas estandarizadas.

\section{MÉTODO \\ PARTICIPANTES}

De los 41 niños examinados inicialmente, se seleccionaron 15 niños mexicanos y 15 niños argentinos, de ambos sexos (19 varones y 11 niñas), con una media de edad de 6 años y 9 meses, que estaban finalizando el primer año de escolaridad primaria. Se excluyeron 11 niños por no cumplir con los criterios de inclusión establecidos: ausencia de dificultades de aprendizaje detectadas y reportadas por padres o maestros y la aplicación del principio alfabético en la escritura (Ball \& Blachman, 1991).

Los niños concurrían a instituciones escolares públicas y privadas que atienden a niños de diferentes niveles socioeconómicos (NSE), caracterizados por el nivel educacional de los padres. La selección de las escuelas tuvo en cuenta el tipo de población que atendían. Para ello se utilizó una adaptación del cuestionario sociodemográfico de Alva y Arboleda (1990) que proporcionó datos acerca del nivel de escolaridad y ocupación de los padres, así como también datos adicionales de los niños (salud, bilingüismo, convivencia, número de hermanos, orden de nacimiento, entre otros).

El NSE medio fue conformado por niños que asistían a escuelas privadas del Distrito Federal de México y a escuelas públicas dependientes de la Universidad Nacional de La Plata (Argentina). En ambos casos, los padres contaban con 16 o más años de estudio. 
El NSE bajo se integró por niños que concurrían a escuelas públicas de la periferia de las mencionadas localidades, cuyos padres tenían no más de 9 años de estudios.

Se controló que participara la misma cantidad de niños mexicanos y argentinos de NSE bajo y medio: 8 niños de NSE medio y 7 de NSE bajo en cada grupo muestral.

\section{INSTRUMENTOS Y MEDICIONES}

\section{1.- PRUebas de EscRituRa espontánea y al dic- TADO DE ORACIONES Y TEXTOS}

Estas pruebas fueron especialmente elaboradas para este trabajo y permitieron observar la cantidad y tipo de segmentaciones lexicales no convencionales que aparecen en las producciones escritas espontáneas y al dictado de oraciones y textos.

Se elaboraron oraciones al dictado, compuestas por palabras que aparecen en ambos países con una frecuencia similar en las edades consideradas, y que incluyen diversos tipos de ítemes, cuya estructura interna corresponde a los criterios elaborados por $\mathrm{Di}$ Tullio (1999).

Ante la inexistencia de una publicación unificada sobre la frecuencia léxica en América Latina, se tomaron en cuenta los trabajos de Alva Canto y Hernández Padilla (2001) para México, y los de Signorini (1999) y Diuk (2003) para Argentina.

El texto al dictado fue extraído de la Prueba de Evaluación de los Procesos Lectores PROLEC (Cuetos, Rodríguez \& Ruano, 1996) y adaptado de tal forma que incluyera palabras con estructura interna de diversa complejidad.

Las pruebas de escrituras se describen en la Evaluación de la Separación entre Palabras en la Escritura (ver Anexo).

\section{2.- Pruebas de Lectura y escrituRA}

Se utilizó el Test de Lectura y de Escritura en Español (LEE - Defior Citoler et al., 2006) que permite obtener el nivel de desem- peño en lectura y escritura. Esta prueba evalúa principalmente el reconocimiento de palabras y la comprensión lectora, a través de actividades de lectura de palabras y pseudopalabras, de frases y de textos en niños de primero a cuarto año de la escolaridad primaria. Asimismo, incluye tareas de dictado de palabras y pseudopalabras. Permite clasificar los resultados obtenidos en tres categorías: nivel muy competente (por encima del percentil 70), nivel competente (percentiles entre 30 y 70) y con dificultad (percentil por debajo de 30). Ha sido estandarizada utilizando muestras de niños argentinos y españoles. Los procedimientos usados por los autores para medir la confiabilidad del LEE fueron test-retest $(r=.50$ a $.88, p<.05)$ y división en dos mitades $(r=.55$ a .70 , $p<.05)$. En cuanto a la validez convergente informan correlaciones positivas y significativas en Lectura de Palabras del LEE y del PROLEC $(r=.512, p<.01)$. Para la evaluación de los niños mexicanos, las pruebas fueron adaptadas lingüísticamente, utilizando términos equivalentes extraídos del estudio transversal de niños de 5 a 12 años realizado por Alva Canto y Hernández Padilla (2001). Al no contar con baremos mexicanos, la comparación de los resultados obtenidos se realizó en base a los puntajes directos del test de referencia.

\section{3.- PRUeba de AMPLITUd de Vocabulario}

Se administró la estandarización argentina del Subtest de Vocabulario de la Escala de Inteligencia WISC III (Wechsler, 1994). No se dispone de una adaptación mexicana de la misma prueba. No obstante resultan similares los ítemes de vocabulario del WISC III respecto del WISC R, utilizado en ese país. A través de esta prueba se obtiene el caudal de vocabulario medido a partir de las puntuaciones ponderadas del subtest. En los manuales se informa que la confiabilidad del mismo se calculó a través del método de división en dos mitades, corregidos por la fórmula Spearman-Brown, obteniendo un valor promedio en las distintas edades de 
.87 , del coeficiente de estabilidad por el método test-retest, $r=.80$ para $6 / 7$ años, y de la concordancia entre examinadores, $r=.92$, cuando se realizó el análisis por grupo de edad.

Respecto de la validez, se indican datos sobre el análisis factorial y la correlación del WISC III con otras escalas de inteligencia como el WISC-R. En el caso de la Prueba de Vocabulario, se halló una correlación entre los puntajes de estas dos pruebas de inteligencia de .77. La comparación de los resultados se realizó en base a los puntajes directos del subtest de referencia, dado que no se dispone de baremos mexicanos.

\section{Procedimiento y Diseño}

Se llevó a cabo un estudio no experimental, transeccional y correlacional (Hernández Sampieri, Fernández Collado \& Baptista Lucio, 2006).

Los niños fueron examinados individualmente, en los establecimientos escolares a los que concurrían en el caso de la ciudad de La Plata (Argentina) y en las instalaciones del Laboratorio de Infantes de la Facultad de Psicología de la Universidad Nacional de México (UNAM). Se solicitó el consentimiento de los padres para la evaluación y se garantizó la confidencialidad de los datos. Las administraciones de las pruebas fueron realizadas por evaluadores altamente capacitados en el tema, en dos sesiones de $60 \mathrm{mi}$ nutos de duración cada una; se controló que la nacionalidad del evaluador coincidiera con la de los niños examinados.

En el análisis lingüístico de las segmentaciones lexicales no convencionales, considerando la insuficiencia de clasificaciones anteriores para dar cuenta de la totalidad de fenómenos observados, se ha tomado como unidad de análisis la noción gramatical de formante morfológico (Di Tullio, 1999). Esa noción es definida por la autora como la unidad mínima que consta de una forma fonética y de un significado. Los formantes pueden ser léxicos o gramaticales. Los léxicos tienen un significado semántico y constituyen clases abiertas de palabras, tales como los sustantivos, adjetivos, adverbios y verbos. Los gramaticales tienen un significado gramatical no léxico y conforman clases cerradas, tales como preposiciones, afijos $\mathrm{y}$ formantes de compuestos (aun-que; por-que; si-no). Asimismo, los formantes se pueden combinar o aparecer como palabras independientes.

Según los criterios mencionados, las palabras pueden conformar las siguientes categorías:

- Palabra Simple: está integrada por un único formante morfológico independiente.

- Palabra Compuesta: contiene dos formantes que pueden aparecer cada uno como una palabra independiente (por ejemplo: cuentagotas; girasol).

- Palabra Compleja: tiene dos o más formantes pero sólo uno de ellos puede ser una palabra independiente, es decir, contiene afijos (por ejemplo: gota-s; got-era; util-iza-ble).

En primer lugar se clasificaron los errores observados en la escritura de oraciones y textos espontáneos y al dictado de la totalidad del corpus obtenido, constituido por 150 oraciones y 30 textos espontáneos y 150 oraciones y 30 textos al dictado. Un $20 \%$ de esa producción fue revisada por dos examinadores expertos, para controlar la existencia de coincidencia en la identificación de los fenómenos bajo estudio.

En segundo lugar, el análisis del desempeño en las pruebas de vocabulario, de lectura y escritura de palabras y pseudopalabras, y de comprensión de frases y textos, fue realizado utilizando los puntajes directos, dado que sólo se dispone de baremos argentinos.

En tercer lugar, las respuestas obtenidas fueron volcadas a una base de datos. Se calcularon estadísticos descriptivos e inferenciales: frecuencia y porcentaje de niños con niveles competentes en el LEE según na- 
cionalidad; diferencias significativas en ese desempeño según nacionalidad; frecuencia y porcentaje de niños con distintos niveles de desempeño en vocabulario; frecuencia y porcentaje de niños que cometieron errores de segmentación en el grupo total y según nacionalidad; frecuencia, medias, desvíos estándar y diferencias significativas de la totalidad de las hipo e hipersegmentaciones producidas por el grupo total y según nacionalidad; frecuencia de los subtipos de segmentaciones no convencionales según la clasificación utilizada.

Finalmente se calcularon las correlaciones entre desempeño en lectura y escritura, nivel de vocabulario y separaciones no convencionales.

\section{Resultados}

1.- LA SEPARACIÓN ENTRE PALABRAS EN LA ESCRITURA. CRITERIOS UTILIZADOS PARA SU CLASIFICACIÓN

La caracterización en detalle del conjunto de las separaciones no convencionales que aparecieron en el corpus examinado, no resultó posible a partir de las clasificaciones utilizadas en otros trabajos, basadas en la diferenciación exclusiva de elementos con y sin significado pleno.

La clasificación propuesta en este trabajo posibilitó incluir todas las manifestaciones de las segmentaciones no convencionales en las escrituras infantiles y delimitar tipos y subtipos de hipo e hipersegmentaciones. La descripción de las categorías propuestas se presenta en la Figura 1 (Querejeta, 2011).

2.- CARACterísticas del NIVEL DE LECTURA, ESCRITURA Y VOCABULARIO EN NIÑOS MEXICANOS Y ARGENTINOS

La mayoría de los 30 niños participantes resolvió adecuadamente las distintas pruebas del LEE, obteniendo puntajes directos que corresponden a niveles competentes de desempeño. Las diferencias de porcentaje según nacionalidad alcanzaron significación estadística en el caso de la lectura de palabras y pseudopalabras (ver Tabla 1).

En el análisis de las puntuaciones en lectura y escritura según la nacionalidad de los niños, se utilizó una prueba estadística no paramétrica ( $U$ de Mann Whitney). Aparecieron diferencias que alcanzaron significación estadística en las siguientes variables: Lectura de palabras: $U=39.5, p=.002$; Lectura de pseudopalabras: $U=26.5, p=.005$; Comprensión de textos $U=56.5, p=.02$. En las dos primeras subpruebas, las puntuaciones más altas las obtuvieron los niños mexicanos y en comprensión de textos, los argentinos. En cuanto al desempeño en la Prueba de Vocabulario (WISC III), el 79.9\% de los niños obtuvo puntajes directos cercanos a la puntuación total máxima, de modo tal que no resultó discriminativa en esos niveles de edad.

\section{3.- LAS SEPARACIONES NO CONVENCIONALES ENTRE PALABRAS EN LA ESCRITURA}

\section{1.- CANTIDAD Y PORCENTAJE DE NIÑOS MEXICANOS Y ARGENTINOS QUE COMETIERON SEPARACIONES NO CONVENCIONALES EN LA ESCRITURA}

En la mayoría de los sujetos evaluados se observaron segmentaciones erróneas, siendo diferencial su aparición según se tratara de escritura espontánea o al dictado. En la escritura espontánea, el porcentaje de separaciones no convencionales en las oraciones fue igual a $56.7 \%$, mientras que en los textos ascendió al 70\%. En la escritura al dictado, esos porcentajes ascendieron al $86.7 \%$ tanto para la escritura de oraciones como de textos.

En el análisis de los resultados según la nacionalidad de los niños no se encontraron diferencias significativas entre ambos grupos (ver Tabla 2).

\section{2.- TIPO Y CANTIDAD DE SEPARACIONES NO CON- VENCIONALES QUE COMETIERON LOS NIÑOS MEXICA- NOS Y ARGENTINOS}

En la muestra total se encontró una preponderancia de las hiposegmentaciones, tanto en la escritura espontánea como al dic- 
tado. Sólo apareció un número mayor de hipersegmentaciones en la escritura al dictado de textos. La aparición de segmentaciones no convencionales mixtas fue menor.

Los errores cometidos aparecieron más frecuentemente en la escritura al dictado en ambos grupos (ver Tabla 3).

Complementariamente se calcularon las medias y los desvíos estándar de las hipo e hipersegmentaciones ${ }^{2}$, considerando sólo las producciones de los niños de ambas nacionalidades que presentaron estos fenómenos. En la mayoría de las tareas de escritura aparecieron diferencias en los valores absolutos en el número de errores, que fueron mayores en el caso de los niños argentinos. Cabe señalar la excepción en las hiposegmentaciones en textos espontáneos y las hipersegmentaciones en textos al dictado cometidas por los niños mexicanos, en las cuales la magnitud de la media y del desvío estándar de los errores cometidos fue mayor, dando cuenta de una gran variabilidad en este grupo. Sin embargo, no se encontraron diferencias significativas al comparar las medias según la nacionalidad de los niños (ver Tablas 4 y 5).

\section{3.- FRECUENCIA DE LOS SUBTIPOS DE HIPO E HI- PERSEGMENTACIONES SEGÚN NACIONALIDAD DE LOS NIÑOS}

Las frecuencias y los porcentajes de los subtipos de errores que presentaron los dos grupos por nacionalidad, se han calculado para la escritura al dictado, teniendo en cuenta la posibilidad de comparación de las producciones analizadas (ver Tablas 6 y 7).

En ambos grupos, tanto en el caso de las hiposegmentaciones como de las hipersegmentaciones, aparecieron errores en la mayoría de los tipos y subtipos considerados. No obstante el error más frecuente correspondió al subtipo $a$, es decir, uniones de un formante gramatical y un formante lexical y

2 Se omitieron las segmentaciones mixtas dado el número reducido de errores de ese tipo. separaciones de un segmento de la palabra que en otro contexto constituye una palabra gramatical. Sin embargo, en las hipersegmentaciones cometidas en la escritura al dictado de textos en los niños mexicanos, el más frecuente fue el subtipo $b$ (separaciones de un segmento de la palabra que constituye en otro contexto una palabra con significado lexical).

4.- LAS CORRELACIONES ENTRE EL DESEMPEÑO EN LECTURA Y ESCRITURA, EL NIVEL DE VOCABULARIO Y LAS SEPARACIONES NO CONVENCIONALES EN LA ESCRITURA

Se calcularon las correlaciones (Rho de Spearman) entre la puntuación según número de segmentaciones erróneas (hiposegmentaciones e hipersegmentaciones) y los puntajes directos en lectura y escritura de palabras (ver Tablas 8 y 9). Aparecieron valores bajos en algunos casos, $r_{\mathrm{S}}=-.52 \mathrm{a}-.57$, que explican del $27 \%$ al $32 \%$ de la variancia. No se encontraron correlaciones que alcanzaran significación estadística entre las segmentaciones erróneas en la escritura y el nivel de vocabulario.

\section{DisCUSIÓN Y CONCLUSIONES}

En este estudio se analizaron las segmentaciones no convencionales que producen niños argentinos y mexicanos que finalizaban el primer año de escolaridad primaria. $\mathrm{Su}$ interés reside en la posibilidad de especificar las características que asumen los fenómenos estudiados y su posible relación con otras variables en diferentes poblaciones, cuya instrucción no es idéntica, en relación con las expectativas de logro para ese período escolar. Para ello, se analizaron las producciones en escritura espontánea y al dictado de oraciones y textos, los desempeños en lectura y escritura y la amplitud de vocabulario.

Entre los resultados de este estudio se destaca, en primer lugar, la utilidad de la clasificación propuesta en trabajos anteriores (Querejeta, 2011) que toma como unidad de 
análisis la noción gramatical de formante morfológico. Ello posibilita el abordaje lingüístico de todas las ocurrencias de hipo e hipersegmentaciones que producen los niños examinados, de modo tal que resulta más adecuado que los realizados en términos de palabra-función y palabra-contenido ( $\mathrm{Fe}$ rreiro \& Pontecorvo, 1996; Tolchinsky, 2006), puesto que muchos de los errores observados no pueden ser incluidos en esas únicas categorías.

En segundo lugar, que los fenómenos examinados aparecen en sujetos con diferente nivel en el desempeño de la lectura de palabras y pseudopalabras. Los niños mexicanos obtuvieron puntajes más altos, lo que pone en evidencia un mayor dominio del principio alfabético con respecto a los argentinos. Esta situación es esperable teniendo en cuenta que en México existen objetivos de enseñanza que implican una intervención pedagógica sistemática, conforme a métodos de tipo fónico, que posibilitan el aprendizaje del lenguaje escrito desde el nivel preescolar. Los métodos derivados de la psicogénesis, predominantes en Argentina, esperan pasivamente un cambio en los niños respecto de sus conceptualizaciones sobre la escritura, que difícilmente ocurra sin una intervención sistemática que apunte a incrementar las habilidades fonológicas (Marder, 2008).

No obstante la importancia del reconocimiento de las letras y la ejercitación de las habilidades fonológicas en las etapas iniciales de la lectoescritura, es preciso aclarar que el dominio del principio alfabético no resulta suficiente para comprender y producir textos progresivamente más complejos, así como respetar las convenciones ortográficas respecto de la separación entre palabras, tal como se ha puesto en evidencia en este estudio.

En tercer lugar, en cuanto a la frecuencia de aparición de las segmentaciones no convencionales entre palabras, las hiposegmentaciones resultaron mayores que las hipersegmentaciones, tal como se ha señalado en otros estudios (Baéz, 1999; Correa \& Dockrell, 2007; Ferreiro \& Pontecorvo, 1996; Tolchinsky, 2006). Asimismo las caracterís- ticas que asumieron resultaron semejantes en los dos grupos, con independencia del método de enseñanza utilizado. Por un lado, las puntuaciones promedio de los errores cometidos no arrojaron diferencias significativas según la nacionalidad de los niños. Por otro, el tipo de errores cometido resultó similar: si bien las hiposegmentaciones aparecieron mayoritariamente por la unión de un formante lexical y uno gramatical, en otros casos se unieron sólo formantes lexicales o gramaticales, que ponen de manifiesto nuevamente la utilidad de la categorización propuesta.

En cuarto lugar, que las segmentaciones lexicales no convencionales pudieron observarse con mayor frecuencia en la escritura al dictado, particularmente cuando se trató de textos, cuyas demandas cognitivas resultan más complejas respecto de la escritura de palabras y oraciones, para lectores y escritores principiantes. Una de las interpretaciones posibles de las diferencias según se trate de la escritura espontánea o al dictado, es que las primeras están relacionadas con las palabras que los niños conocen y consecuentemente codifican cuando se les solicita que escriban lo que quieran. En las oraciones y textos al dictado aparecen segmentaciones erróneas aunque se trate de palabras de uso frecuente en el lenguaje oral espontáneo. Estas palabras incluidas en la escritura al dictado tienen una estructura interna más compleja.

Finalmente, las correlaciones de los fenómenos bajo estudio con tareas de desempeño en lectura y escritura y amplitud de vocabulario, resultaron bajas.

Por el contrario, Correa y Dockrell (2007) en un estudio de niños de primero, segundo y tercer año de escolaridad, encontraron que las hipo e hipersegmentaciones se relacionaron con niveles bajos de lectura, de conciencia morfológica, de vocabulario y capacidad verbal, examinadas estas últimas con el WISC III. Inversamente, en este estudio, esos errores aparecieron en los niños de primer año, con diferentes niveles de lectura y con niveles de vocabulario superiores a la media.

En relación con ello, es posible sostener, con las limitaciones del caso teniendo en cuenta el número de niños examinados, que 
las segmentaciones erróneas van más allá de los desempeños en esas variables y aparecen como una etapa evolutiva en el dominio progresivo de la reflexión y manipulación conciente sobre los ítemes lexicales. Posiblemente, su alta frecuencia en el nivel de escolaridad examinado se deba a la sobrecarga cognitiva que recae en la codificación de cada ítem lexical, que dificulta la reflexión lexical, con la consecuencia de tratar algunos de ellos como si fueran un continuo. Las competencias lexicales avanzarían a partir de la consolidación de las representaciones ortográficas que acompañan a las representaciones fonológicas, sintácticas y semánticas de las palabras. De acuerdo a Erhi (1997, 1998) se produciría una amalgama entre la ortografía, pronunciación y significado, almacenada en el léxico mental, no sólo en el caso de la lectura sino también en el de la escritura.
Resulta de interés señalar que no siempre las segmentaciones lexicales no convencionales son detectadas en la escuela y su persistencia, de no mediar estrategias de intervención adecuadas, pueden tener repercusiones ulteriores en la producción textual que corresponde a niveles más avanzados del trayecto formativo.

La prosecución de esta investigación prevé indagar las relaciones que guardan los resultados obtenidos con el nivel de vocabulario empleando una prueba más sensible en este nivel de edad. Asimismo, resultaría necesario contar con estudios longitudinales en distintos tramos del trayecto formativo, en un número mayor de niños, que posibiliten corroborar los resultados encontrados y esclarecer la evolución de los fenómenos examinados en ese nivel escolar y a lo largo del trayecto formativo. 
FIGURA 1

CLASIFICACIÓN DE LAS SEPARACIONES NO CONVENCIONALES ENTRE PALABRAS

\begin{tabular}{|c|c|c|}
\hline $\begin{array}{l}\text { Separación no } \\
\text { convencional }\end{array}$ & Tipo de error & Ejemplos \\
\hline $\begin{array}{l}\text { Hiposegmentaciones } \\
\text { (uniones no conven- } \\
\text { cionales) }\end{array}$ & 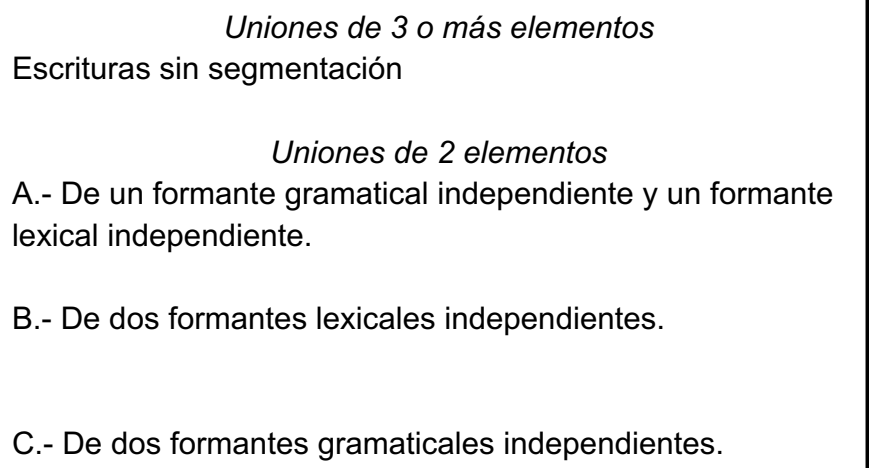 & $\begin{array}{l}\text { ISusanaestabacontenta/ } \\
\text { /fueronaverlatele/ } \\
\text { /derepente/ } \\
\text { /sefue/ } \\
\text { /estabantodas/ } \\
\text { /gatocomiendo/ } \\
\text { /enla/ lalos/ }\end{array}$ \\
\hline $\begin{array}{l}\text { Hipersegmentaciones } \\
\text { (separaciones no } \\
\text { convencionales) }\end{array}$ & $\begin{array}{l}\text { A.- Una palabra es tratada como si estuviera integrada por } \\
\text { formantes independientes, cuando alguna de sus partes } \\
\text { constituye en otro contexto una palabra de significado gra- } \\
\text { matical. } \\
\text { B.- Una palabra es tratada como si estuviera integrada por } \\
\text { formantes independientes, cuando alguna de sus partes } \\
\text { constituye en otro contexto una palabra con significado lexi- } \\
\text { cal. } \\
\text { C.- Una palabra es tratada como si fueran dos palabras sim- } \\
\text { ples, una con significado gramatical y otra lexical. } \\
\text { D.- Una palabra es tratada como dos palabras simples de } \\
\text { significado lexical. } \\
\text { E.- Una palabra es tratada como si estuviera integrada por } \\
\text { tres formantes, dos de ellos gramaticales independientes. } \\
\text { F.- Separaciones silábicas arbitrarias, de las que no se infiere } \\
\text { una reflexión sobre la estructura interna de la palabra. }\end{array}$ & $\begin{array}{l}\text { en-tonces } \\
\text { con-tento } \\
\text { oye-ron } \\
\text { fue-ron } \\
\text { comiendo-se } \\
\text { a-probaba. } \\
\text { Jose-fina } \\
\text { rapida-mente } \\
\text { en-con-traron } \\
\text { en-contra-ron } \\
\text { lim-piaba } \\
\text { fi-esta }\end{array}$ \\
\hline Mixtas & $\begin{array}{l}\text { Combinan características de las hipo e hipersegmentaciones: } \\
\text { entre dos palabras se produce una unión y una separación } \\
\text { no convencional. }\end{array}$ & $\begin{array}{l}\text { loa-pagan } \\
\text { esta-bacontenta }\end{array}$ \\
\hline
\end{tabular}


La separación entre palabras en la escritura infantil

TABLA 1

FRECUENCIA Y PORCENTAJE DE NIÑOS CON NIVELES COMPETENTES EN LAS TAREAS DEL LEE SEGÚN SU NACIONALIDAD

\begin{tabular}{|c|c|c|c|}
\hline Pruebas & Mexicanos & Argentinos & Total \\
\hline Lectura de palabras* & $13(43.3 \%)$ & $6(20 \%)$ & $19(63.3 \%)$ \\
\hline Lectura de pseudopalabras* & $13(43.3 \%)$ & $6(20 \%)$ & $19(63.3 \%)$ \\
\hline Comprensión de palabras y frases & $8(26.6 \%)$ & $9(30 \%)$ & $17(56.7 \%)$ \\
\hline Comprensión de textos & $11(36.6 \%)$ & $12(40.3 \%)$ & $23(76.7 \%)$ \\
\hline Prosodia & $14(46.6 \%)$ & $10(33.3 \%)$ & $24(80 \%)$ \\
\hline Escritura de palabras & $9(30 \%)$ & $8(26.6 \%)$ & $17(56.7 \%)$ \\
\hline Escritura de pseudopalabras & $11(36.6 \%)$ & $9(30 \%)$ & $20(66.7 \%)$ \\
\hline
\end{tabular}

${ }^{*} p<.05$

TABLA 2

PORCENTAJES DE NIÑOS QUE COMETIERON SEPARACIONES NO CONVENCIONALES EN LAS TAREAS DE ESCRITURA SEGÚN NACIONALIDAD

\begin{tabular}{|l|c|c|c|c|}
\hline \multicolumn{1}{|c|}{ Niños } & $\begin{array}{c}\text { Espontánea } \\
\text { oraciones }\end{array}$ & Espontánea textos & Al dictado oraciones & Al dictado textos \\
\hline Mexicanos & 9 & 9 & 14 & $93.3 \%$ \\
Argentinos & $60 \%$ & $60 \%$ & 13 & $86.7 \%$ \\
& 8 & 12 & $80 \%$ & 12 \\
& $53.3 \%$ & $80 \%$ & & $86.7 \%$ \\
\hline
\end{tabular}


TABLA 3

TIPO Y CANTIDAD DE SEPARACIONES NO CONVENCIONALES EN LAS PRUEBAS DE ESCRITURA SEGÚN LA NACIONALIDAD DE LOS NIÑOS

\begin{tabular}{|c|c|c|c|c|c|c|}
\hline \multirow{2}{*}{ Escritura } & \multicolumn{2}{|c|}{ Hiposegmentaciones } & \multicolumn{2}{|c|}{ Hipersegmentaciones } & \multicolumn{2}{|c|}{ Mixtas } \\
\hline & $\mathrm{M}$ & A & $\mathrm{M}$ & A & M & A \\
\hline Oraciones espontáneas & 18 & 13 & 4 & 3 & 3 & 1 \\
\hline Textos espontáneos & 44 & 25 & 8 & 19 & 3 & 1 \\
\hline Oraciones al dictado & 26 & 27 & 13 & 31 & 7 & 4 \\
\hline Textos al dictado & 20 & 35 & 33 & 27 & 11 & 0 \\
\hline
\end{tabular}

Nota:

M: Mexicanos

A: Argentinos

TABLA 4

MEDIAS Y DESVÍOS DE LAS HIPOSEGMENTACIONES EN LAS TAREAS DE ESCRITURA DE LOS NIÑOS QUE PRODUJERON ESTE TIPO DE ERRORES

\begin{tabular}{|l|cc|cc|cc|}
\hline \multirow{2}{*}{ Hiposegmentaciones } & \multicolumn{2}{|c|}{ Grupo Total } & \multicolumn{2}{c|}{ Mexicanos } & \multicolumn{3}{c|}{ Argentinos } \\
& $M$ & $D E$ & $M$ & $D E$ & $M$ & \\
\hline & & & & & \\
& & & & & \\
Oraciones espontáneas & 2.07 & 1.66 & 2.00 & 2.00 & 2.17 & 1.16 \\
Textos espontáneos & 3.63 & 3.13 & 5.50 & 3.96 & 2.27 & 1.34 \\
Oraciones al dictado & 2.21 & 1.25 & 2.17 & 1.26 & 2.25 & 1.28 \\
Textos al dictado & 2.50 & 1.81 & 1.82 & 1.25 & 3.18 & 2.08 \\
\hline
\end{tabular}

TABLA 5

MEDIAS Y DESVÍOS DE LAS HIPERSEGMENTACIONES EN LAS TAREAS DE ESCRITURA DE LOS NIÑOS QUE PRODUJERON ESTE TIPO DE ERRORES

\begin{tabular}{|l|cc|cc|cc|}
\hline \multirow{2}{*}{ Hipersegmentaciones } & \multicolumn{2}{|c|}{ Grupo Total } & \multicolumn{2}{c|}{ Mexicanos } & \multicolumn{2}{c|}{ Argentinos } \\
& $M$ & $D E$ & $M$ & $D E$ & $M$ & $D E$ \\
\hline & & & & & & \\
Oraciones espontáneas & 1.17 & .40 & 1.00 & - & 1.50 & .70 \\
Textos espontáneos & 1.80 & 1.01 & 1.33 & .51 & 2.11 & 1.16 \\
Oraciones al dictado & 2.59 & 1.22 & 2.15 & .98 & 2.82 & 1.32 \\
Textos al dictado & 2.73 & 2.07 & 3.00 & 2.60 & 2.45 & 1.44 \\
\hline
\end{tabular}


TABLA 6

DISTRIBUCIÓN DE LOS SUBTIPOS DE HIPOSEGMENTACIONES EN ESCRITURA AL DICTADO SEGÚN LA NACIONALIDAD

\begin{tabular}{|l|cc|cc|}
\hline \multicolumn{1}{|c|}{ Tipo de errores } & \multicolumn{2}{|c|}{ Escritura al dictado de oraciones } & \multicolumn{2}{|c|}{$\begin{array}{c}\text { Escritura al dictado de textos } \\
\text { M }\end{array}$} \\
\hline $\begin{array}{l}\text { Sin segmentación } \\
\text { Error Subtipo a: Unión de formante lexical y }\end{array}$ & $3(11.5 \%)$ & $8(29.6 \%)$ & - & $2(5.7)$ \\
formante gramatical & $21(80.7 \%)$ & $14(51.8)$ & $19(95 \%)$ & $28(80 \%)$ \\
Error Subtipo $b$ : Unión de formantes lexicales & $2(7.6 \%)$ & $4(14.8 \%)$ & - & $4(11.4 \%)$ \\
$\begin{array}{l}\text { Error Subtipo c: Unión de formantes gramati- } \\
\text { cales }\end{array}$ & - & $1(3.7 \%)$ & $1(5 \%)$ & $1(2.8)$ \\
\hline Total & 26 & 27 & 20 & 35 \\
\end{tabular}

Nota:

M: Mexicanos

A: Argentinos

TABLA 7

DISTRIBUCIÓN DE LOS SUBTIPOS DE HIPERSEGMENTACIONES EN LA ESCRITURA AL DICTADO SEGÚN LA NACIONALIDAD

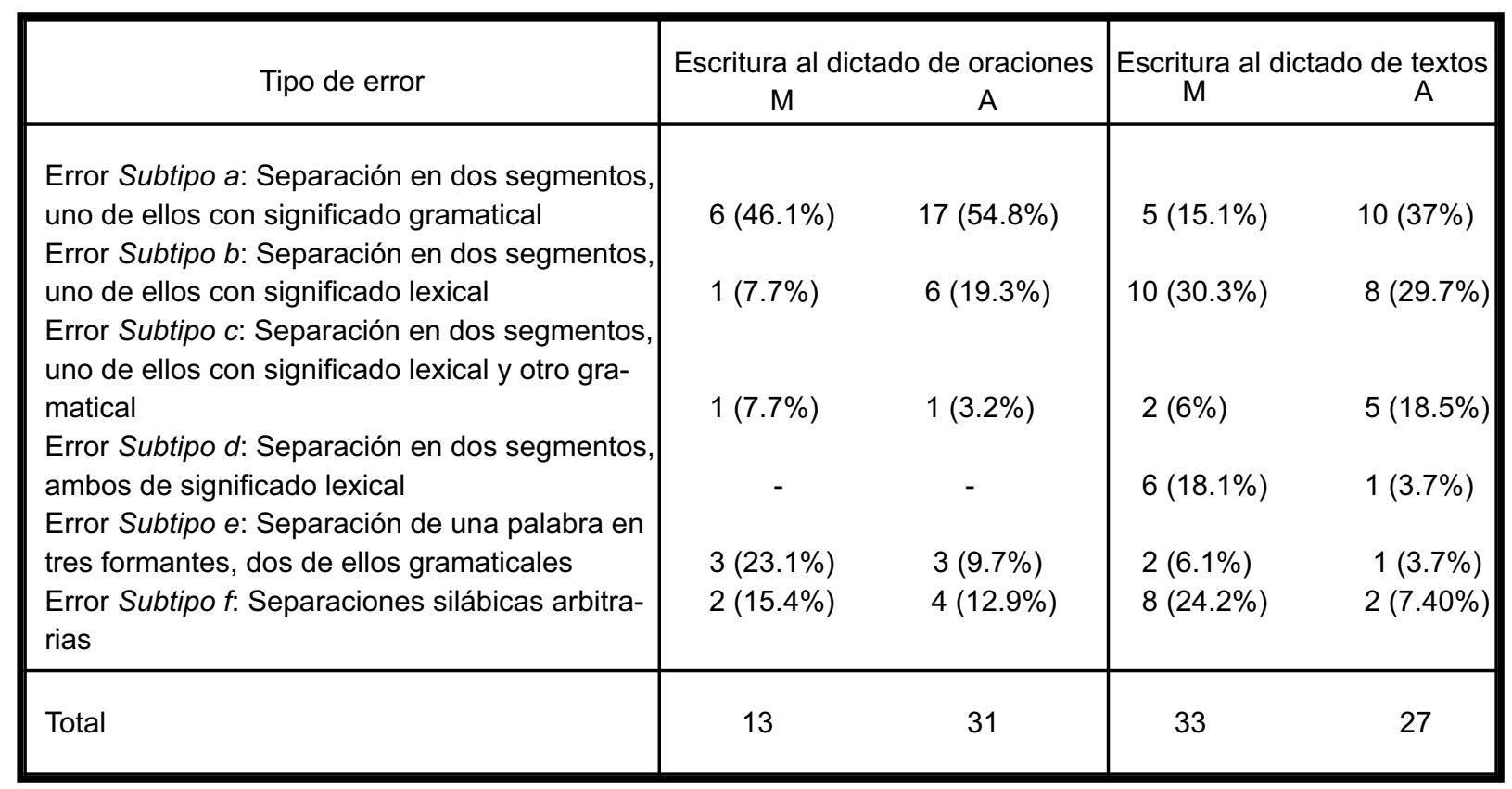

Nota:

M: Mexicanos

A: Argentinos 
TABLA 8

CORRELACIONES ENTRE LAS SEPARACIONES NO CONVENCIONALES EN LA ESCRITURA DE ORACIONES AL DICTADO Y EL DESEMPEÑO EN LECTURA Y ESCRITURA DE PALABRAS Y PSEUDOPALABRAS EN AMBOS GRUPOS DE NIÑOS

\begin{tabular}{|c|c|c|c|c|c|c|c|c|}
\hline \multirow{3}{*}{ Pruebas } & \multicolumn{4}{|c|}{ Hiposegmentaciones } & \multicolumn{4}{|c|}{ Hipersegmentaciones } \\
\hline & \multicolumn{2}{|c|}{ Mexicanos } & \multicolumn{2}{|c|}{ Argentinos } & \multicolumn{2}{|c|}{ Mexicanos } & \multicolumn{2}{|c|}{ Argentinos } \\
\hline & $r_{\mathrm{s}}$ & $p$ & $r_{\mathrm{S}}$ & $p$ & $r_{\mathrm{s}}$ & $p$ & $r_{\mathrm{S}}$ & $p$ \\
\hline Lectura de Palabras & -.22 & .42 & -.47 & .07 & .19 & .47 & -.39 & .14 \\
\hline Lectura de Pseudopalabras & -.19 & .49 & -.41 & .12 & .12 & .65 & -.29 & .29 \\
\hline Escritura de Palabras & -.08 & .76 & -.46 & .07 & -.37 & .17 & $-.52^{*}$ & .04 \\
\hline Escritura de Pseudopalabras & -.16 & .56 & $-.52^{*}$ & .04 & -.14 & .59 & $-.53^{*}$ & .04 \\
\hline
\end{tabular}

${ }^{*} p<.05$

${ }^{* *} p<.01$

TABLA 9

CORRELACIONES ENTRE LAS SEPARACIONES NO CONVENCIONALES EN LA ESCRITURA DE TEXTOS AL DICTADO Y EL DESEMPEÑO EN LECTURA Y ESCRITURA DE PALABRAS Y PSEUDOPALABRAS EN AMBOS GRUPOS DE NIÑOS

\begin{tabular}{|c|c|c|c|c|c|c|c|c|}
\hline \multirow{3}{*}{ Pruebas } & \multicolumn{4}{|c|}{ Hiposegmentaciones } & \multicolumn{4}{|c|}{ Hipersegmentaciones } \\
\hline & \multicolumn{2}{|c|}{ Mexicanos } & \multicolumn{2}{|c|}{ Argentinos } & \multicolumn{2}{|c|}{ Mexicanos } & \multicolumn{2}{|c|}{ Argentinos } \\
\hline & $r_{\mathrm{S}}$ & $p$ & $r_{\mathrm{S}}$ & $p$ & $r_{\mathrm{S}}$ & $p$ & $r_{\mathrm{S}}$ & $p$ \\
\hline Lectura de Palabras & .05 & .83 & -.35 & .19 & -.43 & .10 & -.27 & .33 \\
\hline Lectura de Pseudopalabras & .00 & 1.0 & -.37 & .16 & -.34 & .20 & -.22 & .42 \\
\hline Escritura de Palabras & .37 & .16 & $-.57^{*}$ & .02 & -.25 & .36 & -.36 & .18 \\
\hline Escritura de Pseudopalabras & .13 & .64 & $-.52^{*}$ & .04 & $-.57^{*}$ & .02 & -.29 & .29 \\
\hline
\end{tabular}

${ }^{*} p<.05$

${ }^{* *} p<.01$ 


\section{ANEXO}

\section{EVALUACIÓN DE LA SEPARACIÓN ENTRE PALABRAS EN LA ESCRITURA}

\section{1.- Escritura espontánea}

En todos los niveles escolares se administraron las mismas pruebas de escritura espontánea de oraciones y de textos.

Se solicitó a los niños las siguientes tareas:

- Escribir cinco oraciones.

- Escribir un cuento a partir de reactivos pictóricos, que muestren secuencialmente una historia. Se utilizaron para ello los ítemes "PASO" y "FUEGO", del Subtest Ordenamiento de Historias del WISC III (Wechsler, 1994). Estos ítemes fueron seleccionados del total que compone la prueba en función de pertinencia para elicitar la producción de narrativas y hacer comparable el corpus a obtener.

\section{2.- Escritura al dictado}

Se administraron tareas de escritura al dictado de oraciones y textos.

\subsection{1.- Escritura al dictado de oraciones}

Las oraciones al dictado se elaboraron teniendo en cuenta la frecuencia (Alva Canto \& Hernández Padilla, 2001 para México y Signorini, 1999 y Diuk, 2003 para Argentina) de las palabras incluidas. La cantidad de palabras se incrementó en los distintos niveles escolares. Al dictar las oraciones se tuvo especial cuidado en hacerlo conforme a los cánones de la oralidad, de modo tal que no fueran sugeridas las segmentaciones propias de la escritura.

1.- Susana estaba contenta.

2.- Los nenes encontraron la pelota.

3.- Entonces fueron a ver la tele.

4.- Juan fue a buscar algunas monedas.

5.- De repente, el gato saltó sobre el ratón.

\subsection{2. - Escritura al dictado de textos}

Se seleccionaron textos sobre la misma temática que resultaran familiares a los niños. Se adaptaron de pruebas estandarizadas, Evaluación de los Procesos Lectores - PROLEC (Cuetos, Rodríguez \& Ruano, 1996), de modo tal de incluir palabras con estructura interna de diversa complejidad y de aumentar la cantidad de palabras en los distintos niveles escolares. También en este caso se tuvo especial cuidado en dictarlos conforme a los cánones de la oralidad, de modo tal de no sugerir las segmentaciones propias de la escritura.

Texto incluido: "Era el cumpleaños de Marisa y ahí estaban todas sus amigas esperando que empezara la fiesta. De repente, oyeron un ruido en la cocina. Entraron para ver qué pasaba y encontraron al gato comiendo la torta." 


\section{REFERENCIAS BIBLIOGRÁFICAS}

Adams, M. (1990). Beginning to read: Thinking and learning about print. Cambridge: MIT Press.

Alva Canto, E.A. \& Hernández Padilla, E. (2001). La producción del lenguaje de niños mexicanos. Un estudio transversal de niños de cinco a doce años [The Mexican's children language production. A cross-sectional study of children aged five to twelve years]. México: Universidad Nacional Autónoma de México.

Alva, E.A. \& Arboleda, D. (1990). Análisis de las interacciones verbales en dos grupos de niños preescolares [Analysis of verbal interactions in two groups of preschoolar children]. Trabajo presentado en el IV Congreso Mexicano de Psicología, México D.F.

Báez, M. (1999) La problemática de la segmentación gráfica del texto en palabras: Indagación en niños de sectores rurales [The problem of graph segmentation of text into words: Inquiry into children in rural areas]. Lectura y Vida, Año 20(2), 22-36.

Ball, E.W. \& Blachman, B. (1991). Does phoneme awareness training in kindergarten make a difference in early word recognition and developmental spelling? Reading Research Quarterly, 24, 49-66. doi: 10.1598/R RQ.26.1.3.

Borzone de Manrique, A.M. \& Massone, M.I. (1987). La palabra como unidad de análisis en el desarrollo fonológico [The word as unit of analysis in phonological development]. Manuscrito inédito.

Cook Moats, L. (2010). Speech to print. Language essencials for teachers. Baltimore: Paul Brookes Publishing.

Correa, J. (2005). Escrita e segmentação lexical não-convencional na criança [Writing and unconventional lexical segmentation in childhood]. Trabajo presentado en el I Congresso Latino-Americano de Psicologia. São Paulo.

Correa, J. \& Dockrell, J. (2007). Unconventional word segmentation in Brazilian children's early text production. Reading and Writing, 20, 815-831. doi: 10.1007/s11145-006-90493.

Cuetos, F., Rodríguez, B. \& Ruano, E. (1996). Evaluación de los Procesos Lectores: PROLEC [Evaluation of Reading Processes: PROLEC]. Madrid: TEA Ediciones.

Defior Citoler, S., Fonseca, L., Gottheil, B., Aldrey, A., Jiménez Fernández, G., Pujals, M., Rosa, G. \& Serrano Chica, F.D. (2006). LEE. Test de Lectura y Escritura en Español [LEE. Test of Reading and Writing in Spanish]. Buenos Aires: Paidós.

DGCE. (2008). Diseño curricular para la educación primaria. Primer ciclo [Curriculum for primary education. First cycle]. Buenos Aires: Dirección General de Cultura y Educación de la Provincia de Buenos Aires.

Di Tullio, A. (1999). Manual de gramática del español [Manual of Spanish grammar]. Buenos Aires: Edicial.

Diuk, B. (2003). El proceso de aprendizaje de la lectura y la escritura en sectores urbanomarginados: Un estudio cognitivo [Reading and writting learning process in marginalized urban minorities: Acognitve study]. Tesis Doctoral no publicada. Facultad de Humanidades y Ciencias de la Educación de la Universidad Nacional de La Plata, La Plata.

Erhi, L. (1997). Learning to read and learning to spell are one and the same, almost. En C. Perfetti, L. Riebeen, \& M. Fayor (Eds.), Learning to spell (pp. 237-269). Hillsdale, NJ: Erlbaum.

Erhi, L. (1998). Grapheme-phoneme knowledge is essential for learning to read words in 
English. En J.L. Metsala \& L.C. Erhi (Eds.), Word recognition in beginnig literacy (pp. 340). Mahwah, NJ: Erlbaum.

Ferreiro, E. \& Pontecorvo, C. (1996). Los límites entre las palabras [The boundaries between words]. En E. Ferreiro, C. Pontecorvo, N. Ribeiro \& I. García, Caperucita roja aprende a escribir. Estudios psicolingüisticos en tres lenguas (pp. 45-73). Barcelona: Gedisa.

Ferreiro, E. \& Teberosky, A. (1979). Los sistemas de escritura en el desarrollo del niño [Writing systems in child development]. México: Siglo Veintiuno Editores.

Gombert, J.E. (1990). Développement métalinguistique et langage écrit [Metalinguistic development and written language]. En E. Gombert (Ed.), Le développement métalinguistique (pp. 197-225). París: Presses Universitaires du France.

Hernández Sampieri, M.C., Fernández Collado, C. \& Baptista Lucio, P. (2006). Metodología de la investigación [Research methodology]. México: McGraw-Hill.

Linares Rivas, A. (2000). Hacia una competencia ortográfica integral [Towards an integral orthographical competition]. San Cristóbal, Venezuela: Universidad de los Andes Táchira, Laboratorio de Investigaciones Lingüísticas.

Marder, S. (2008). Impacto de un programa de alfabetización temprana en niños de sectores urbano-marginales [Impact of an early literacy intervention program in children of poor urban sectors]. Tesis Doctoral no publicada. Facultad de Psicología de la Universidad Nacional de La Plata, La Plata.

Molinari, C. \& Corral, A. (2008). La escritura en la alfabetización inicial. Producir en grupos en la escuela y el jardín [Writing in early literacy. Producing in groups in the school and the preschool]. La Plata: Dirección Ge- neral de Cultura y Educación de la Provincia de Buenos Aires.

Piacente, T. (2005). Trastornos de aprendizaje del lenguaje escrito [Disabilities of written language learning]. Facultad de Humanidades y Ciencias de la Educación de la UNLP (mimeo).

Querejeta, M. (2011). La separación entre palabras en la escritura infantil de oraciones $y$ textos [The separation between words in children's writing of sentences and texts]. Tesis Doctoral no publicada. Facultad de Psicología de la Universidad Nacional de La Plata, La Plata.

RAE. \& ASALE. (2010). Ortografía de la lengua española [Orthography of the Spanish language]. Madrid: Real Academia Española.

SEP. (2011a). Programas de estudio 2011. Guía para el maestro. Educación básica. Preescolar [Curriculum 2011. Teacher guide. Basic education. Preschool]. México: Secretaría de Educación Pública.

SEP. (2011b). Programas de estudio 2011. Guía para el maestro. Educación básica. Primaria. Primer grado [Curriculum 2011. Teacher guide. Basic education. Primary. First grade]. México: Secretaría de Educación Pública.

Signorini, A. (1999). Del habla a la escritura: El procesamiento fonológico en la lectura inicial [From speech to writing: The phonological processing in early reading] Tesis Doctoral no publicada. Facultad de Humanidades y Ciencias de la Educación de la Universidad Nacional de La Plata, La Plata.

Snow, C.E. \& Juel, C. (2005). Theaching children to read: What do we know about how to do it? En M.J. Snowling \& Ch. Hulme (Eds.), The science of reading: A handbook (pp. 501520). Malden, MA: Blackwell Publishing.

Tolchinsky, L. (2006). The emergence of writing. En C. Mac Arthur, S. Graham \& J. Fitzgerald 
(Eds.), Handbook of writing research (pp. 8395). New York: The Guilford Press.
Wechsler, D. (1994). Test de Inteligencia para Niños: WISC III [Intelligence Test for Children: WISC III]. Buenos Aires: Paidós.

Instituto de Psicología Facultad de Psicología Universidad Nacional de La Plata (UNLP) Consejo Nacional de Investigaciones Científicas y Técnicas (CONICET)

La Plata, Prov. de Buenos Aires República Argentina Laboratorio de Infantes Facultad de Psicología Universidad Nacional Autónoma de México (UNAM)

México

Fecha de recepción: 10 de mayo de 2012 Fecha de aceptación: 9 de mayo de 2013 